\title{
Musculoskeletal ultrasound imaging - integration with the biopsychosocial model
}

Dr Sue Innes ${ }^{(1)}$, Professor Jo Jackson ${ }^{(1)}$

1. School of Sport, Rehabilitation and Exercise Sciences.

University of Essex.

Wivenhoe Park

Colchester

CO4 3SQ

United Kingdom

Sue Innes email: inness@essex.ac.uk

Jo Jackson email: jo.jackson@essex.ac.uk

\section{Abstract}

\section{Background and Method}

Musculoskeletal ultrasound imaging, (MSKUSI) has become a popular imaging modality in recent years and is being utilised by a variety of professions in clinical environments beyond radiology departments. A previously published study exploring physiotherapists' interests and use of MSKUSI in practice included in-depth interviews of participants, $(n=11)$. The data from this qualitative study was analysed thematically and five themes were identified; one was 'Application of the biopsychosocial model', the basis of this paper. 


\section{Results}

The theme, 'Application of the biopsychosocial model' drew together three categories of analysed data: clinical reasoning, professional variance and communication opportunity. 'Clinical reasoning' reflected the participants' value on subjective assessment information and the integration of ultrasound imaging with physical examination findings. 'Professional variance' observed the scanning processes undertaken by physiotherapists tended to vary from other professionals, the physiotherapists perceived their use of dynamic imaging was greater, in particular scanning in symptom provoking positions. Another variance observed was the style of communication that physiotherapists used when scanning, this was categorised as a 'communication opportunity' as it represented an event that could be utilised to promote patient understanding of their presentation, link imaging findings to proposed management and enhance compliance with rehabilitation strategies. Participants emphasised their responsibility when communicating with patients to avoid language that could promote unhelpful behaviour, e.g. catastrophisation.

\section{Conclusion}

Participants placed value on integrating musculoskeletal ultrasound imaging into the biopsychosocial model, further research to explore the impact of this approach on patients' clinical outcomes and reported experiences is required.

\section{Keywords}


Musculoskeletal ultrasound, physiotherapy, biopsychosocial assessment, communication, clinical reasoning.

\section{Introduction}

Musculoskeletal ultrasound imaging (MSKUSI) is an imaging modality used by a variety of professionals in diverse clinical environments, $(1,2,3,4)$. The professionals attracted to this modality include physiotherapists and several aspects of physiotherapists' engagement with MSKUSI have been reported, $(5,6,7)$. These studies have researched topics such as education accessed and barriers to modality utilisation but have been restricted by their data collection tools - questionnaires. The integration of imaging knowledge with clinical assessment and the clinical reasoning processes physiotherapists undertake has not been explored and reflects a gap in the evidence base. This paper is the second of two publications that report the findings of research aimed to evaluate physiotherapists' interests and clinical application of MSKUSI. The first paper, (ref) presents the research methodology and a summary of the study's results. This second paper focuses on one research element; the link between physiotherapists' clinical application of MSKUSI and the biopsychosocial model, $(8,9)$.

Musculoskeletal physiotherapists' professional education and practice is influenced by the widespread application of the biopsychosocial model including knowledge of non-nociceptive pain and complex pain states, $(8,9,10)$. In contrast, literature 
exploring MSKUSI is dominated by the orthodox medical model of tissue-based pathology $(1,2,4)$. Published musculoskeletal sonography case studies typically include a brief history of the patient's presentation, investigations and interventions that precede the ultrasound assessment but tend not to explore the reasoning that enables the imaging findings to be integrated with clinical assessment. Imaging findings that can be regarded as the key structural causes of patients' symptoms are highlighted and whilst 'incidental' and 'age appropriate' findings are commonly reported, the link between tissue changes identified by ultrasound and pain is emphasised $(1,5)$. This model of tissue-based pathology is reflected in traditional ultrasound training and therefore the practice of clinicians including radiologists and sonographers $(1,16,17)$. Physiotherapists' practice based integration of ultrasound imaging with their understanding of pain states and knowledge of musculoskeletal medicine has not yet been included in literature.

The overall aim of this study was to explore why physiotherapists are interested in MSKUSI and what are its clinical roles for this professional group?

\section{Methods}

\section{Research Design}

This mixed-methods ultrasound based research has been reported in a previous publication, (11). A questionnaire was developed and was distributed to access a relevant population of participants with three distribution methods: by hand at a physiotherapy conference, (Association of Chartered Physiotherapists in Orthopaedic Medicine and Injection Therapy), by email invitation for consenting 
members of a specialised professional group interested in ultrasound, (ElectroPhysical Agents and Diagnostic Ultrasound network) and by a professional online discussion forum led by the United Kingdom's professional body, the Chartered Society of Physiotherapy. The questionnaire was accompanied by a participant information sheet that detailed the study's aims and researcher's background. The intended roles of the survey included accessing physiotherapists with an interest in MSKUSI, collecting background data about the physiotherapists including their work environment and educational history in MSKUSI, enabling a purposive sampling strategy for the second part of the study and gaining consent from physiotherapists who would be willing to be interviewed.

The questionnaire generated 75 responses, 34 of these respondents reported that they used MSKUSI in practice, the nature of the clinical practice for the questionnaire respondents and the interview participants has been presented in Table 1. A purposive sampling strategy selected the interview participants ensuring they were informationally representative from pre-selected criteria determined by the questionnaire; the 11 interview participants all used MSKUSI, worked in a range of clinical settings, had undertaken varying ultrasound related education and had reported factors impacting their ability to use the imaging modality. The questionnaire data also facilitated the development of a topic-guide for the second part of the study.

The questionnaire data also facilitated the development of a topic guide for the second part of the study. The topic guide ensured interviews explored key concepts relevant to the research question $(12,13)$. Participants were facilitated to describe their experiences with MSKUSI including education accessed, support or barriers experienced, current clinical application and their vision for using the modality in the 
future. 11 participants were interviewed at their place of work by $X X$, the interviews' duration was each approximately 1 hour, field notes were taken and interviews were digitally recorded and transcribed verbatim.

The primary researcher was a female doctoral student $(X X)$ and physiotherapist with no clinical scanning experience. The researcher's professional background and familiarity with musculoskeletal terminology enabled exploration of topics relevant to the research question during the in-depth discussions.

Table 1: Nature of clinical practice for respondents (note some respondents worked in more than one environment).

\begin{tabular}{|l|l|l|l|}
\hline $\begin{array}{l}\text { Professional } \\
\text { Environment }\end{array}$ & $\begin{array}{l}\text { Environment } \\
\text { reported by all } \\
\text { respondents to } \\
\text { questionnaire } \\
(\mathbf{n = 7 5 )}\end{array}$ & $\begin{array}{l}\text { Environment } \\
\text { reported by } \\
\text { scanning } \\
\text { respondents to } \\
\text { questionnaire } \\
(\mathbf{n = 3 4 )}\end{array}$ & $\begin{array}{l}\text { Environment for } \\
\text { interview } \\
\text { participants } \\
(\mathbf{n}=\mathbf{1 1})\end{array}$ \\
\hline NHS & 43 & 19 & 8 \\
\hline Private practice & 31 & 15 & 3 \\
\hline Private hospital & 4 & 2 & 0 \\
\hline $\begin{array}{l}\text { Sports team or } \\
\text { institute }\end{array}$ & 8 & 3 & 1 \\
\hline Research & 10 & 5 & 1 \\
\hline
\end{tabular}

\section{Interview Data Analysis}

The transcribed data were analysed thematically, this process is well suited to exploratory studies that require inductive reasoning whereby the investigator's analysis facilitates identification of codes and themes without being framed by 
preconceptions, (14). Thematic analysis approaches to exploratory research questions tend to differ to confirmatory questions in that the codes and analytic categories are not predetermined. Whilst confirmatory studies may test hypotheses, exploratory studies do not and may generate hypotheses.

Six steps framed the thematic analysis process, (Braun and Clarke 2006):

1. Familiarising yourself with your data

2. Generating initial codes

3. Searching for themes

4. Reviewing themes

5. Defining and naming themes

6. Producing the report.

The first step, 'familiarising yourself with your data' commenced at an early stage in the research process, during the interviews and continued during transcription. Denaturalised transcription facilitated analysis as it responded to the study's aims of exploring the data's information content rather than the conversation style, (Oliver et al 2005). Transcription was followed by reading and rereading of the transcribed interviews and ensured data familiarity.

In this study, there were no pre-identified codes or theme, coding was guided by the principles documented by Saldaňa,(25). The data-condensation and analysis process was facilitated by MAXQDA, (Version 11, Verbi Software) enabling 
formation of subcategories, categories and themes. Data coding and theme formation were verified by a second researcher, $(X)$ who was independent from the data collection process

\section{Interview Results}

Five themes were identified, they were named to reflect the essence of their content:

1. Professional skill set - physiotherapists' suitability for MSKUSI

2. Factors that have impacted physiotherapists' ability to use MSKUSI

3. Physiotherapists' Motivation to Use Ultrasound - Improving Patient Focused Care

4. Quality Assurance Strategies

5. Application of Biopsychosocial Model

The five themes and their professional relevance have been discussed in a previous publication, (11).The fifth theme, 'Application of Biopsychosocial Model' will now be presented in more detail.

\section{Theme: Application of Biopsychosocial Model:}

The component codes that informed the sub-categories and categories of this theme have been summarised in Table 2. Example quotations from participants have been included to support the results and evidence the rich data obtained from the semistructured interviews. 
Table 2: Code grouping and formation of categories in theme 5 - Application of Biopsychosocial Model

\begin{tabular}{|c|c|c|}
\hline Codes & Subcategories & Categories \\
\hline Subjective informs scan & \multirow[t]{2}{*}{ Subjective assessment } & \multirow[t]{4}{*}{ Clinical reasoning } \\
\hline Yellow flags & & \\
\hline Verify clinical assessment & \multirow[t]{2}{*}{ Clinical assessment } & \\
\hline Guided by clinical assessment & & \\
\hline Image quality & \multirow[t]{2}{*}{$\begin{array}{l}\text { Imaging - different } \\
\text { professionals }\end{array}$} & \multirow[t]{5}{*}{$\begin{array}{l}\text { Professional } \\
\text { Variance }\end{array}$} \\
\hline Lack of context & & \\
\hline Physiotherapist communication & \multirow[t]{3}{*}{$\begin{array}{l}\text { Communication - } \\
\text { different professionals }\end{array}$} & \\
\hline Radiologist's communication & & \\
\hline Absence of communication & & \\
\hline Tissue based pain & \multirow{2}{*}{$\begin{array}{l}\text { Communication in } \\
\text { presence of abnormal } \\
\text { tissue }\end{array}$} & \multirow[t]{7}{*}{$\begin{array}{l}\text { Communication } \\
\text { opportunity }\end{array}$} \\
\hline Communicate pathology & & \\
\hline Non-tissue based pain & \multirow{5}{*}{$\begin{array}{l}\text { Communication in } \\
\text { presence of normal } \\
\text { findings }\end{array}$} & \\
\hline Patient information when normal & & \\
\hline Chronic pain prevention & & \\
\hline Requirement for rehabilitation & & \\
\hline Role of Reassurance & & \\
\hline
\end{tabular}




\section{Category: Clinical Reasoning}

\section{Subcategory: Subjective Assessment}

Participants reported that scanning was a component of a wider examination and not an event to occur in isolation. Several, who had unlimited access to an ultrasound system were able to choose precisely when to scan patients and the role of MSKUSI in the assessment. Other participants had limited machine access, so organised scanning lists as an add-on to their normal assessment. Some reported part of their working week was in radiology where the scan was the focal examination process, these clinicians still supported the scan with information gained by questioning or brief clinical examination procedures. These assessment procedures informed the scanning process by providing context; the subjective assessment, (full or modified) was identified as the key means of obtaining context and also highlighted psychosocial features that may have relevancy.

'I would think of it while you are taking the subjective based on what they say and you think, maybe the mechanism of injury or something in the history makes you think, maybe a scan will be useful' (PT3)

Several participants identified specific components of the subjective assessment that influenced the scanning procedure or their overall clinical decision making. The two components that were referred to on several occasions were the activities patients reported as pain provocative or problematic, (clinicians generally called these aggravating and easing factors) and the identification of yellow flags. Participants commented that detailed information gained about provocative activities during the subjective facilitated targeted scanning procedures: 
'and we have actually got them in the position with the probe and shown them, 'look that pinches' and they go 'ow, that's my pain'.' (PT2)

The term 'yellow flags' relates to psychological, social and environmental factors that could increase the likelihood of disability and originally were used with reference to low back pain, (15). Psychological factors include unhelpful beliefs about pain and injury that result in behaviours such as extended rest or movement avoidance. Social and environmental factors include difficulties with claims or compensation, perceptions of a lack of support from the work place and overly protective family members, (16). The flag classification system has extended in recent years and has become more complex; some authors refer to an assortment of flag colours that relate to psychological, work and environmental factors. Blue flags have been proposed to describe work-related issues, black flags relate to practical obstacles such as insurance systems and orange flags symbolise psychiatric conditions including clinical depression, (17). This complicated colour coding is not consistent in the literature and was not used by the participants so, for the purpose of this study, the term 'yellow flags' relates to all psychological, social and environmental considerations.

Yellow flags were discussed by participants in three distinct ways and supported by clinical examples, for instance their potential to sustain a painful presentation and cause pain amplification, $(18,19)$ was raised by one participant:

If you have examined somebody and asked the right questions and think that they are somebody who is perhaps, higher risk or in medium risk bracket for developing disability secondary to their pain problem: the injury was at work and they have already been off and not been back and things are disproportionate, and none of it 
makes any great deal of sense in terms of these wide receptive fields and there are neurological sensations, nothing really appears mechanical with it.' (PT8)

Several participants' comments drew on their knowledge of pain physiology and observed, that for some patients with psychosocial markers alongside persistent pain, imaging findings were unlikely to fully explain symptoms, $(15,17,18)$.

The second link between yellow flags and scanning found was a strong sense of professional responsibility; the clinicians did not want the imaging process to increase the likelihood of patients developing preventable chronic presentations, (20, 21). There were several examples when clinicians emphasised they were careful not to promote any yellow flag related beliefs or behaviour by poorly considered communication:

I have got that responsible position of using ultrasound in a way that does not then make the patient scared, catastrophising concern about findings that are not relevant.' (PT5)

The third view point regarding the application of yellow flag identification and MSKUSI also reflected professional responsibility. Participants demonstrated an awareness of professional errors, in particular the risk of over-reliance on psychological contributions to a patient's presentation, (22). These clinicians observed that patients who appeared to demonstrate excessive pain behaviours or 
other yellow flags warranted thorough investigations as their symptoms could be predominantly nociceptive in origin:

'She did not look like she was going to get off crutches, after 4 weeks she was still on crutches, my friend said 'can you scan this patient, I think she is a malingerer?'. Scanned the patient, found a twelve centimetre calf tear, aponeuritic tear medial calf, typical tennis leg.' (PT4)

\section{Subcategory: Clinical Assessment}

The material coded into subcategory 'clinical assessment' provided evidence of advanced clinical reasoning processes as the participants described systems to link MSKUSI with the clinical assessment. Clinical reasoning skills were described as enabling and were utilised in two subtly different ways in relation to the clinical assessment's outcome. Firstly, reasoning supported the process of verifying findings from the clinical examination and secondly, as a facilitator of the scanning process; the participants reported they wanted to respond to clinical findings instead of following a standard scanning protocol.

The prevalent opinion was that ultrasound verified clinical examination findings, it followed them, it did not precede them and clinical reasoning processes drew on the strengths of both strategies to reach a diagnosis. One participant ended her discussion on this subject emphatically stating, 'I won't treat on a scan'. (PT2) 
The second clinical reasoning application was the participants' adaption of scanning protocols in response to the clinical assessment, many evidenced confident clinical reasoning that enabled this responsive scanning. One participant described a comfortable process of moving between scanning and physical testing:

I may actually get the ultrasound machine out straight away because while I am conducting the whole clinical assessment and you end up sometimes jumping between the two.' (PT10)

\section{Category: Professional Variance}

\section{Subcategory: Communication - different professionals}

Variation in communication practice between scanning physiotherapists and other scanning professionals was raised by several participants. Participants placed significance on the opportunity MSKUSI provided to communicate with patients. They observed that communication following musculoskeletal scans conducted by scanning clinicians who are not physiotherapists typically focused on confirming the process by which the referring clinician will receive the scan report and did not necessarily include many details of the scan findings themselves:

'Whereas typical sonographers or radiologists from the department, they say, 'this is what we found, we will let your consultant or GP know, they will know what to do' (PT9) 


\section{Subcategory: Imaging Different Professionals}

Participants observed that several elements of their scanning practice varied from the scanning that they had observed by some other scanning professionals. Some elements of scanning highlighted that were not always seen to be valued by other professions included: the guidance of subjective information, deviation from protocols when indicated, responsiveness to patients' aggravating and easing factors and communication alongside dynamic scanning to optimise the education opportunity.

Participants were keen to highlight that other professions brought their own unique skill sets and training to MSKUSI and emphasised their radiologist colleagues were undertaking tasks that were reliant on their medical expertise. One participant was supporting a sonographer with his MSKUSI training and she observed the advantages his extensive clinical experience had on his ability to optimise his ultrasound image:

'he's learning the musculoskeletal pathology and yes, the musculoskeletal clinical reasoning. But his handling and his pictures are beautiful' (PT2)

\section{Category: Communication Opportunity}

Communication to patients was a topic that many participants discussed extensively and emphasised as playing a key role. 


\section{Subcategory: Communication in the Presence of Abnormal Tissue}

Participants were unanimous that communication in the presence of abnormal tissue should be factual to ensure imaging findings are explained. They also repeatedly highlighted this explanation of tissue-based findings must be placed in context and that pain presentations may not be fully explained by the scan's findings. The contextualisation of scan findings has been summarised by the following participant:

'As a physio, because I have an understanding of orthopaedic medicine and I have an understanding of this pathology and the management of it, because I work in a triage service, so I share those with the patients' (PT1)

Communication in the presence of abnormal tissue was reported as an opportunity that should be respected; the participants placed great value on explaining tissuebased findings, relating them to the patient's symptoms and ensuring that irrelevant scan findings were not discussed inappropriately. :

'we know that it is very difficult to pick all the influences and pain is multifactorial by its nature..... I am really aware that you shouldn't be seen as a physio who is using sonography, be re-enforcing those things I have already seen that in situations where: 'all of this must be really sore or this must be awful'. Because, we know that there is not really a direct correlation between pathology and symptoms, so we should not be saying "this is what you are feeling', there is a risk of that.' (PT8)

Participants suggested the scanning experience increased patients' belief and trust in the physiotherapists' message, in particular when the scan verified the findings and explanation that accompanied the clinical examination: 
'I think that it definitely helps with understanding and education is an important part of trying to dictate compliance, I think there might be that. I think it gives confidence, therapeutic alliance, believing someone, being credible'. (PT 5)

This participant highlighted the possible link between credibility and compliance, suggesting patients who believe in their physiotherapist's opinion and are provided with education may be more compliant with recommended management than patients of physiotherapists who do not scan. These links were expressed in subtly different ways by many participants, the following participant reported his patients appeared satisfied with a verified diagnosis and this facilitated self-management:

'... they have their scans and that tells us what is wrong and that is what cures it' (PT 3)

Researcher: 'telling them what is wrong is what cures it?'

'I think so. The magic of naming isn't it? Patients do say, 'I just want to know what is wrong. ....giving the patients something they can understand and something they can see...... helps to reinforce that message of self-management most of the time.' (PT 3)

\section{Subcategory: Communication in the Presence of Normal Findings}

Many of the participants were keen to emphasise that imaging that revealed normal tissue was still a communication opportunity and could represent a therapeutic event: 
'because if you can reassure somebody 'that things look OK really we can't see anything necessary too wrong'. It is a really powerful message to somebody who is in that kind of situation' (PT8)

The evidence base exploring the role of information and communication in management of pain is extensive and includes issues raised by the participants: causes of chronic pain $(9,23)$, prevention and management of pain, $(10,16,24)$ and the role of reassurance, $(25,26)$. Communication providing education has been related to improved patient self-management for unexplained pain syndromes; a number of studies have concluded that cognitive reassurance (information and explanation) are associated with improved outcomes when compared with effective reassurance, (empathy and rapport), $(25,26)$. A majority of participants conveyed the value they placed on the opportunity to inform and educate in the scanning room.

All of the participants reported the opinion that scanning related education and communication are valuable. Some provided specific examples that reflected their view on the impact of this communication including one participant's comments relating to the management of a challenging patient:

'Just to get them on board really.... I had a right stroppy lad the other week, a 25 year old....... I can see that if he carries on like that, I will scan him just to go, 'look, there isn't fluid, this is not torn, this is here, your bones look pristine', cause I am certain, clinically from his exam, there will be no clinical reason to scan him, but to get him on board, it might be a useful thing.' (PT 2) 


\section{Discussion}

Thematic analysis of interview data revealed the participants integrated the biopsychosocial model into their application of MSKUSI. Existing literature emphasises the dominance of the biopsychosocial model in contemporary physiotherapy practice, $(8,9,10)$ but has not directly linked physiotherapists' use of ultrasound to this model.

Confident integration of ultrasound imaging into the participants' practice was dependent on a full or partial subjective assessment; whilst referral for ultrasound imaging should always include a clear clinical question (29) it is evident that all of these physiotherapist scanning clinicians wanted more patient information to inform their imaging. Similarly, scanning was further guided by a full or modified clinical assessment, with both being tested and verified by the other. The integration of all assessment findings was facilitated by participants' understanding of the biopsychosocial model. Clinical reasoning and applied knowledge of psychological, social and emotional factors that may contribute to patients' presentations were all considered in the imaging interaction. This integration of biological (tissue-based) assessment with psychological and social factors has not been explored in publications to date and represents a significant research opportunity. Further research directions that have emerged from this study, include clinical reasoning underpinning differential diagnosis testing and whether professional background influences the pre-scanning patient interaction or imaging protocol followed. 
Professional variations are inevitable as professions will have contrasting experiences in training undertaken prior to engaging in MSKUSI,(29, 33,34,35). It may be possible that greater transparency regarding these variations may foster inter-disciplinary collaborations and peer learning. Acknowledgement of professional variation may facilitate inter-professional learning, this has yet to be explored in the field of musculoskeletal sonography but evidence from other healthcare settings suggest inter-professional learning can enhance collaboration and communication between professions, $(36,37)$.

The biopsychosocial framework appeared to underpin the participants' approach to MSKUSI related communication, many elements reported align well with existing literature linking the biopsychosocial model to communication and education. Participants placed value on the style of communication to prevent patients developing unhelpful beliefs that could perpetuate pain presentations, (21) and highlighted the opportunity offered by the scanning appointment to enhance therapeutic alliance, (30). The judicious selection of terms used to explain ultrasound findings to prevent catastrophisation aligns with evidence that links inappropriate imaging and imprudent reporting of findings to patients with poor clinical outcomes, $(38,39)$. To date, no publications have explored the impact of the vocabulary and terms selected when communicating ultrasound findings to patients, but this study's participants applied principles that have been documented extensively in the assessment of low back pain and chronic pain syndromes, (38, 39). In addition, exploration of scanning physiotherapists' views on correct and incorrect patient selection for ultrasound assessment in Point of Care and Radiology departments may provide interesting data as evidence suggests imaging modalities 
are over-used for some musculoskeletal presentations. Unjustified X-Rays for nonspecific mechanical low back pain may contribute to a poor treatment outcome, (39) and it is possible that imprudent ultrasound imaging has the same effect. Selection of patients for MSKUSI in Point of Care clinical settings contrasts with radiology and it is unknown if this integrated Point of Care assessment influences patient selection for imaging or clinical outcomes. Whilst participants responded to psychosocial findings, they also expressed a responsibility to investigate all possible biological contributions to pain and were able to place the orthodox medical model of pain in a contemporary multi-factorial framework, $(9,31,32)$. Literature reporting research exploring communication and its contribution to the MSKUSI process is extremely limited but provides preliminary indications that patients value this interaction, (27, 28). There are many elements that warrant further exploration including the impact of scanning related information on patients' trust in their therapist, patients' compliance with rehabilitation and the effect of MSKUSI related education on further management requirements.

\section{Reflexivity}

In qualitative research, the role of the researcher must be considered as their personal experiences and knowledge will influence the entire research process, (40). The significance of the primary researcher's profession warrants consideration as it could be argued that as a physiotherapist, the researcher would choose to present the profession in an unjustifiably positive way. There is a viewpoint that a neutral stance is required to enable the researcher to obtain data that is not biased and to conduct a fair analysis. Qualitative analysts however argue that the researcher's 
position is integral to the research process and the presentation of any study should include a review of this position, $(41,42)$. The primary researcher's position as a physiotherapist has influenced the research process in several ways most notably enabling access to participants and crucially, subject specific knowledge has ensured rich data were collected at the interview stage. Extensive experience in the musculoskeletal specialism and familiarity with professional terms facilitated the semi-structured interviews to progress without pauses for clarification or lack of understanding.

Reflexivity was maintained throughout the entire research process including data analysis. During this process, researchers should attend to the data whilst acknowledging their own influences, biases and assumptions, (43). The analysis of the research data, in particular, the interview data was highly dependent on the primary researcher's ability to interpret the participants' terminology whilst ensuring personal viewpoints did not underpin the analysis.

The semi-structured interviews have provided a novel insight into the links participants made between their physiotherapy assessment skills and MSKUSI, it is proposed that this data was accessible because of the primary researcher's professional background.

\section{Strengths and Limitations}

Methodological quality must be considered and there are some constraints within this study including the impact of the questionnaire's distribution methods and the interview recruitment strategy. Interview participant recruitment was informed by a purposive sampling strategy, this ensured that participants represented specific 
criteria as reported in the questionnaire. These criteria included diversity of clinical and educational backgrounds and representation of the factors that had impacted individuals' engagement with MSKUSI. It is acknowledged that these parameters affect the transferability of the study but that other recruitment strategies add rigour. All of the selected interview participants were regularly scanning and could therefore contribute their perspectives on the role of MSKUSI for physiotherapists and their educational experiences. Recruitment strategies were also strengthened by the inductive thematic analysis process which ensured data saturation was achieved, it was noted that the final interviews conducted did not require the generation of new codes or themes, (44). The interview method used was face to face and took place in work places distributed throughout much of England. Whilst telephone interviews would have required less time for travel, the face to face encounters facilitated detailed exploration of topics and interview-interviewee interaction that would have been difficult to replicate on a telephone. Member checking was not formally included and would have further strengthened the analysis process.

\section{Conclusion}

This component of the study presented in this paper provides insight into use of MSKUSI by physiotherapists, which was reported to be informed and embedded in the biopsychosocial model. The value of communication during and following the ultrasound examination was emphasised by participants. They reported that communication related to imaging enhances patient education, patients' understanding of their presentation and compliance in self-management. Further 
research is needed to explore physiotherapists' use of MSKUSI, topics could include impact on patient satisfaction, understanding and clinical outcomes, also the influence of MSKUSI on patient pathways and resource management.

\section{Ethical Approval}

Ethical approval was awarded by Ethics Committee, Faculty of Science and Health, $\mathrm{X}$, reference number: 13006

No conflicts of interest to declare.

\section{Funding Sources}

This research did not receive any specific grant from funding agencies in the public, commercial, or not-for-profit sectors. 


\section{References}

1. McNally EG. The development and clinical applications of musculoskeletal ultrasound. Skeletal Radiol. 2011;40(9):1223-31.

2. Deimel, G.W., Jelsing, E.J. \& Hall, M.M. Musculoskeletal Ultrasound in Physical Medicine and Rehabilitation. Curr Phys Med Rehabil Rep 2013: 1: 38. https://doi.org/10.1007/s40141-012-0003-9

3. Edwards H. Special Feature Let 's all jump on the ultrasound bandwagon : further debate on the use of ultrasound. Ultrasound. 2010;18:4-7.

4. Patil P, Dasgupta B. Role of diagnostic ultrasound in the assessment of musculoskeletal diseases. Ther Adv Musculoskelet Dis. 2012;4(5):341-55.

5. Potter CL, Cairns MC, Stokes M. Use of ultrasound imaging by physiotherapists : A pilot study to survey use, skills and training. Man Ther. 2012;17(1):39-46. Available from: http://dx.doi.org/10.1016/j.math.2011.08.005 6. Ellis R, De Jong R, Bassett S, Helsby J, Stokes M, Cairns M. Exploring the clinical use of ultrasound imaging: A survey of physiotherapists in New Zealand. Musculoskelet Sci Pract. 2018;34:27-37. Available from: http://www.ncbi.nlm.nih.gov/pubmed/29247981

7. Mckiernan S, Chiarelli P, Warren-Forward H. Radiography A survey of diagnostic ultrasound within the physiotherapy profession for the design of future training tools. Radiography. 2011;17(2):121-5. Available from: http://dx.doi.org/10.1016/j.radi.2010.08.003

8. Jones M, Edwards I, Gifford L. Masterclass: Conceptual models for implementing biopsychosocial theory in clinical practice. Man Ther. 2002;7(1):2-9. 
9. Moseley LG, Butler DS. Critical Review Fifteen Years of Explaining Pain: The Past, Present, and Future. J Pain. 2015;16(9):807-13. Available from: http://dx.doi.org/10.1016/j.jpain.2015.05.005

10. Sanders T, Foster NE, Bishop A, Ong BN. Biopsychosocial care and the physiotherapy encounter: physiotherapists' accounts of back pain consultations. BMC Musculoskelet Disord. 2013;14(1):65. Available from: http://bmcmusculoskeletdisord.biomedcentral.com/articles/10.1186/1471-2474-14-65 11. Innes S, Jackson J. Submitted for publication: Musculoskeletal ultrasound imaging - an exploration of physiotherapists' interests and use in practice 12. Gill P, Stewart K, Treasure E, Chadwick (2008) 'Methods of data collection in qualitative research: interviews and focus groups' British Dental Journal 204 (6): 291-295

13. Carter S \& Henderson L (2009) 'Approaches to qualitative data collection in social science. In: Bowling A and Ebrahim S (eds) Handbook of Health Research Methods, Investigation, Measurement and Analysis (215-229) England: Open University Press McGraw Hill

14. Guest G, MacQueen K, Namey E (2012) Applied Thematic Analysis. Thousand Oaks, California. Sage

15. Kendall N, Linton S, Main C (1997) 'Guide to Assessing Psychosocial Yellow Flags in Acute Low Back Pain: Risk Factors for Long-Term Disability and Work Loss'. Accident Rehabilitation and Compensation Insurance Corporation of New Zealand and the National Health Committee, Wellington, New Zealand 16. Gray H \& Howe T (2013) 'Physiotherapists' assessment and management of psychosocial factors (Yellow and Blue Flags) in individuals with back pain' Physical Therapy Reviews 18 (5): 379-394 
17. Nicholas M, Linton S, Watson PJ, Chris J, Main C (2011) 'Early Identification and Management of Psychological Risk Factors ("Yellow Flags") in Patients With Low Back Pain: A Reappraisal' Physical Therapy 91 (5): 737 -753

18. Linton S \& Shaw W (2011) 'Impact of Psychological Factors in the Experience of Pain' Physical Therapy 91 (5): 700-711

19. Wertli M. M, Held U, Lis A, Campell M, Weiser S. Both positive and negative beliefs are important in patients with spine pain: findings from the Occupational and Industrial Orthopaedic Center registry. The Spine Journal. 2018: 18(8):1463-1474. https://doi.org/10.1016/j.spinee.2017.07.166

20. Wheeler L P, Karran E L, Harvie D S. Low back pain. Can we mitigate the inadvertent psycho-behavioural harms of spinal imaging? Australian Journal of General Practice. 2018: 47 (9):610-613

21. Karran EL, Medalian Y, Hillier SL, Moseley GL. 2017. The impact of choosing words carefully: an online investigation into imaging reporting strategies and best practice care for low back pain. PeerJ 5:e4151 https://doi.org/10.7717/peerj.4151 22. Farre A, Rapley T. 2017. The New Old (and Old New) Medical Model: Four Decades Navigating the Biomedical and Psychosocial Understandings of Health and IIIness. Healthcare 2017, 5(4), 88; doi:10.3390/healthcare5040088

23. Nicholas M, Vlaeyen J, Ried, Barke A, Aziz Q, Benoliel R, Cohen M, Evers S, Giamberardino M A, Goebel A, Korwisi B, Perrot S, Svensson P, Wang S-J, Treede R-D. The IASP Taskforce for the Classification of Chronic Pain. The IASP classification of chronic pain for ICD-11, chronic primary pain. Pain. 2019;160(1): 2837

24. Campbell P, Hill JC, Protheroe J, Afolabi EK, Lewis M, Beardmore R, Hay EM, Mallen CD, Bartlam B, Saunders B, van der Windt DA, Jowett S, Foster NE, 
Dunn KM. Keele Aches and Pains Study protocol: validity, acceptability, and feasibility of the Keele STarT MSK tool for subgrouping musculoskeletal patients in primary care. J Pain Res, 2016; vol. 9, 807-818.

25. Traeger AC, Hübscher M, Henschke N, Moseley GL, Lee H, McAuley JH. Effect of Primary Care-Based Education on Reassurance in Patients With Acute Low Back Pain. JAMA Intern Med . 2015;175(5):733. Available from:

http://www.ncbi.nlm.nih.gov/pubmed/25799308

26. Pincus T, Holt N, Vogel S, Underwood M, Savage R, Walsh D, Taylor S (2013) 'Cognitive and affective reassurance and patient outcomes in primary care: a systematic review' Pain 154 (11): 2407-2416

27. Wheeler P, Trust LNHS. What do patients think about diagnostic ultrasound ? A pilot study to investigate patient-perceived benefits with the use of musculoskeletal diagnostic ultrasound in an outpatient clinic setting. Int Musculoskelet Med. 2010;32(2):68-71.

28. Lumsden G, Lucas-Garner K, Sutherland S, Dodenhoff R. Physiotherapists utilizing diagnostic ultrasound in shoulder clinics. How useful do patients find immediate feedback from the scan as part of the management of their problem? Musculoskeletal Care. 2018;16:209-13.

29. British Medical Ultrasound Society, Society and College of Radiographers. Guidelines For Professional Ultrasound Practice Society and College of Radiographers and British Medical Ultraound Society Guidelines for Professional Ultrasound Practice. 2017. Available from:

https://www.bmus.org/static/uploads/resources/SCoR_BMUS_Guidelines_for_Profe ssional_Ultrasound_Practice_Revised_Jan_2018.pdf 
30. Pinto R, Ferreira M, Oliveira V, Franco M, Adams R, Maher C, Ferreira P (2012) 'Patient-centred communication is associated with positive therapeutic alliance: a systematic review' Journal of Physiotherapy 58 (2): 77-87

31. Beneciuk J \& George S (2015) 'Pragmatic Implementation of a Stratified Primary Care Model for Low Back Pain Management in Outpatient Physical Therapy Settings: Two-Phase, Sequential Preliminary Study' Physical Therapy 95 (8): 11201134

32. Moseley LG, Butler DS. Critical Review Fifteen Years of Explaining Pain: The Past, Present, and Future. J Pain. 2015;16(9):807-13. Available from: http://dx.doi.org/10.1016/j.jpain.2015.05.005

33. Royal College of Radiologists. Ultrasound training recommendations for medical and surgical specialties Third edition. 2017. Available from:

https://www.rcr.ac.uk/publication/ultrasound-training-recommendations-medical-andsurgical-specialties-third-edition

34. Faculty of Sport and Exercise Medicine. The SEM Sonographer - Proposed Standard of the Faculty of Sport and Exercise Medicine(UK). 2013. Available from: https://www.fsem.ac.uk/members-area/my-development/ultrasound/ultrasoundguidelines/sonography-in-sem/

35. lagnocco A, Terslev L, Backhaus M, et al Educational recommendations for the conduct, content and format of EULAR musculoskeletal ultrasound Teaching the Teachers Courses RMD Open 2015;1:e000139. doi: 10.1136/rmdopen-2015-000139 36. World Health Organisation. (2010). Framework for action on interprofessional education \& collaborative practice. Retrieved from http://www. who.int/hrh/resources/framework_action/en/index.html 37. Reeves, S., Pelone, F., Hendry, J., Lock, N., Marshall, J., Pillay, L., \& 
Wood, R. (2016). Using a meta-ethnographic approach to explore the nature of facilitation and teaching approaches employed in interprofessional education. Medical Teacher, 38(12), 1221-1228. doi:10.1080/ 0142159X.2016.1210114

38. Flynn TW, Smith B, Chou R (2011) 'Appropriate use of diagnostic imaging in low back pain: a reminder that unnecessary imaging may do as much harm as good.' The Journal Of Orthopaedic And Sports Physical Therapy 41 (11): 838-46

39. Foster N E, Anema J R, Cherkin D, Chou R, Cohen S P, Gross D P, Ferreira P H, Fritz J M, Koes B W, Peul W, Turner J A, Maher C G (2018) 'Prevention and treatment of low back pain: evidence, challenges, and promising directions' The Lancet 391 (10137): 2368-2383 https://doi.org/10.1016/S0140-6736(18)30489-6

40. Lambert C, Jomeen J, McSherry W (2010) 'Reflexivity: A review of the literature in the context of midwifery research' British Journal of Midwifery 18 (5): 321 $-326$

41. Rolfe G (2006) 'Commentary: Encouraging the use of reflexivity in the writing up of qualitative research' International Journal of Therapy and Rehabilitation 13 (5): 215

42. Darawsheh W (2014) 'Reflexivity in research: Promoting rigour, reliability and validity in qualitative research' International Journal of Therapy and Rehabilitation 21 (12): $560-568$

43. Probst B (2015) 'The Eye Regards Itself: Benefits and Challenges of Reflexivity in Qualitative Social Work Research' Social Work Research 39 (1): 37-48 44. Saunders, B., Sim, J., Kingstone, T. et al. Qual Quant (2018) 52: 1893. https://doi.org/10.1007/s11135-017-0574-8 
